\title{
Three-Stage Growth Model of the Natural Diamond of Octahedral Habit
}

Antonyuk, B.P., and Mironov, V.P.

YaGEER\&D CNIGRI, ALROSA Co. Ltd., Chernyshevsky Sh. 7, Mirny, 678170, Sakha (Yakutia), Russia

The investigated objects were the diamonds (about 500 crystals) of octahedral habit having 1 to 8 $\mathrm{mm}$ size from kimberlite pipe Mir (Yakutia). A luminescent tomography was used during the investigations that permits to visualize the diamond internal morphology due to their luminescence in the ultraviolet light (Mironov and Antonyuk, 1994).

The generalized picture of a crystal genesis of natural octahedral habit diamond is represented as successive change of three growth stages. The formation of diamond crystallization centres that are isolated in the central parts of crystals and that usually have "point-like" sizes takes place during the first (initial) stage. Yet, in some diamond crystals it is possible to clearly observe the octahedral crystallites having the size to $0 . n \mathrm{~mm}$ that luminescence in a bright blue light or do not luminescence. Probably, they can be interpreted as $\{111\}$ crystals nuclei grown to visible sizes. The transition from the first to the second (intermediate) growth stage is abrupt. When the crystal attains a definite size (not more than $0 . n \mathrm{~mm}$ in our case) a octähedral growth form becomes unstable and is changed by a combination of octahedron and rounded surface. Such a cardinal change of a diamond growth form is connected probably with the inhomogeneity of overcooling (oversaturation) on the crystal growing surface at a relatively nonequilibral crystallization conditions. According to the existing knowledge (Chernov et al.,1980) the rounded growth front coinsides with an isotherm (isoconcentrate surface) of crystallization.

In an idealized appearance the diamond rounded growth form is shown in Fig.1. Geometrically the shape having such a surface is formed in the result of intercrossing of eight spheres of equal radii and having only one common point. The spherical parts are divided from each other by kinked lines (circular arcs) in the planes $(x y),(x z),(y z)$. The points of kinking are on the exits of cartesian axes. The general symmetry of the shape consisting of eight parts of the spherical surface (rounded "faces") conforms with the diamond crystal class $(\mathrm{m} 3 \mathrm{~m})$. For the crystallographic characteristic of a rounded growth form we shall use "orientation surface" concept (Mokievsky,1983). Each of the eight rounded "faces" is constituted of nonisolated pointwise orientations :111:, :110:, :100:, :hk0:, $: h h l:,: h l l:,: h k l:$. The rounded growth form on the phantom-crystals contained in the diamond octahedrons is always combined with flat faces $\{111\}$. The growth sectors of the octahedral faces differ in red, blue luminescence or no luminescence.The growth sectors of the rounded "faces" have a yellow-green or yellow colour of luminescence .

The change of the diamond crystal form at the intermidiate stage is defined by the change of relative growth rates of octahedral faces and of rounded "faces". According to the rule (Laemmlein, 1948) the convex side of the boundary between the growth sectors is turned to the face the growth of which was becoming relatively slower. We shall illustrate it on the idealized models on condition that the crystal-forming medium symmetry during the whole growth period corresponds to the symmetry of a sphere, i.e. the crystal growth without the habit distortion, according to the Curie principle (crystal and medium symmetries superposition).

If the growth rate of the rounded "faces" in relation to the octahedral faces increases constantly then in the process of growth the rounded form degenerates (Fig.2). As the growth rate of the rounded form raises, the number of pointwise orientations excluded by the kinks of the rounded surface increases. It is represented in the reduction of the deflection depth of the curved line growth zones. In contrast, the octahedral faces spread. At the intermediate stage the contours of the growing $\{111\}$ 
faces are of edge form $<211>$. After the intersection of adjacent octahedral faces the edge form $<211>$ combines with the edges $<110>$. At the end of the second stage faces $\{111\}$ completely close the growth sectors of rounded form at the exit points of cartesian axes restoring status quo, i.e. the octahedral form existed at the first stage of growth. In this case the contours of octahedral faces are formed only by $<110>$ edges. At the third (final) stage such diamond crystals continue entirely the octahedral growth in the conditions nearly close to equilibrant.

The most numerous are the diamond crystals having the internal morphology that is shown in Fig.3. The boundaries behaviour between the sectors represents a variable relative growth rate of contacting octahedron and rounded form faces. At the beginning of the second stage the rounded surface growth speeds up but to the end of the stage their growth relatively slows down. The latter condition is the reason of octahedral orientations appearing on the rounded surface close to the kinks (in connection with an abrupt increase of the deflections depths). The rounded surface is covered by a great number of $\{111\}$ crystallites. The octahedral diamond macrofaces do not practically grow until a newly formed multiapical and stepped surface (gradually getting coarsened) will not "restore" the crystal to its regular octahedral form. After the noted phenomenon of "restoration" the growth of the diamond continues in an ordinary closed octahedral form. The sectors of octahedron of the final growth stage have blue, orange luminescence or no luminescence. In the case of rhythmically-zonal structure of the $\{111\}$ sectors a successive change of zones with blue luminescence by zones with orange luminescence and finally by zones with no luminescence is noted.

The morphology of the investigated $\{111\}$ habit diamonds is readily explained from the PBC (the periodic bond chains) theory viewpoint (Hartman and Perdok, 1955) taking into account the interaction of only the first (closest) neighbour atoms in the diamond lattice, i.e. the $\mathrm{PBC}<110>$. The $\{111\}$ singular atomically flat faces that are characterized by a tangential growth mechanism completely define the diamond form at the first and the third stages of growth. At the second stage of growth the octahedral F-faces combine with atomically rough rounded "faces", formed by nonsingular pointwise orientations $: 110:,: 100:, h k 0:, h h l:,: h l l:,: h k l:$, that grow according to the normal mechanism. The succession of a natural diamond growth form change is according to the pattern: $\{111\} \rightarrow\{111\}+$ rounded form $\rightarrow\{111\}$. The crystal form evolution during the process of growth represents the change of diamond formation conditions from less to more equilibrant. The absence of traces of interruption between the growth stages, despite a sharp change of the form of growth, allows to consider the crystallization as an uninterrupted process of diamond formation of one generation.

There is contrastly exhibited deep connection between the atomic structure of the growing surfaces, the growth mechanisms, and physical properties (photoluminescence, in particular) of growth sectors and zones in the natural diamonds of octahedral habit. A natural diamond displays good mineralogical memory of its growth history.

\section{References}

Chernov, A., et al., 1980, Modern crystallography, vol.3: Nauka, Moscow, p.167-175 (in Russian). Hartman, P., and Perdok, W., 1955, On the relations between structure and morphology of crystals: Acta Crystallografica, 8(1), p.49-52.

Laemmlein, G., 1948, Sectorial structure of a crystal: Izd. AN SSSR, Moscow-Leningrad, p.1-39 (in Russian).

Mironov, V., and Antonyuk, B., 1994, Distribution of the luminescent centres in yakutian diamonds: Archiwum mineralogiczne, 50(2), p.3 -10.

Mokievsky, V., 1983, Morphology of crystals: Nedra, Leningrad, p.50-61 (in Russian). 


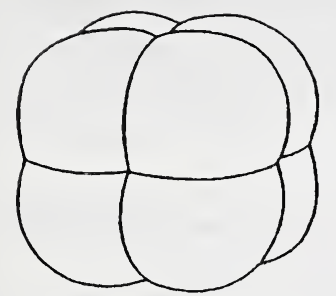

(a)
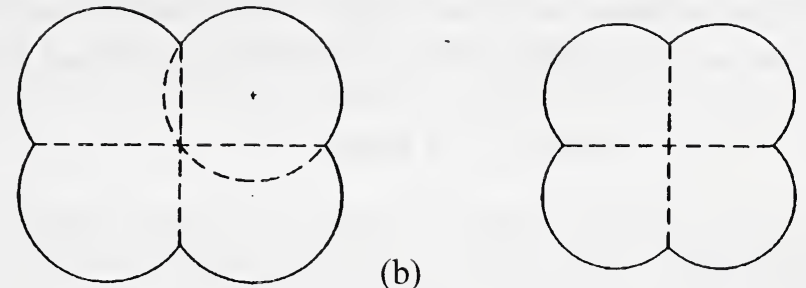

(b)

(c)

Fig.1. A rounded form of the natural diamond growth determined on the phantom-crystals (in reality it is always in combination with the octahedral faces): (a) axonometric view; (b), (c) central sections parallel to (110) and (100).
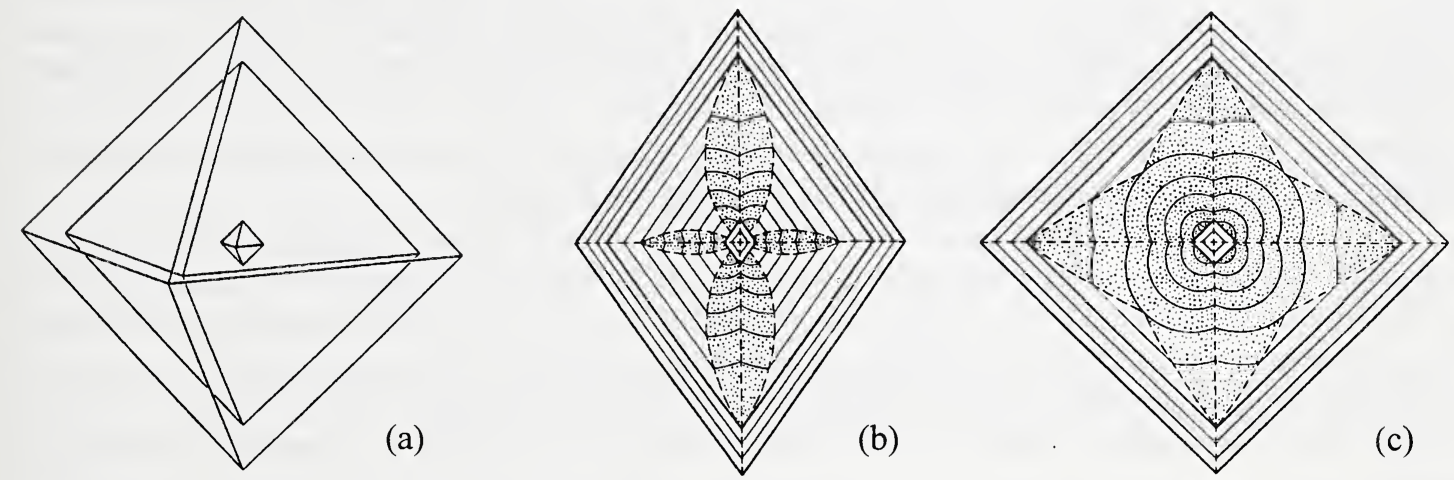

Fig.2. Internal morphology model of a natural diamond of octahedral habit (growth rate of the rounded form at the second stage increases constantly): (a) axonometric view; (b), (c) central sections parallel to (110) and (100). Sectors of rounded form "faces" growth are shown by points. Bold lines are the crystal contours at the end of the growth stages; thin lines are the boundaries between the growth zones; broken lines are the boundaries between the growth sectors.
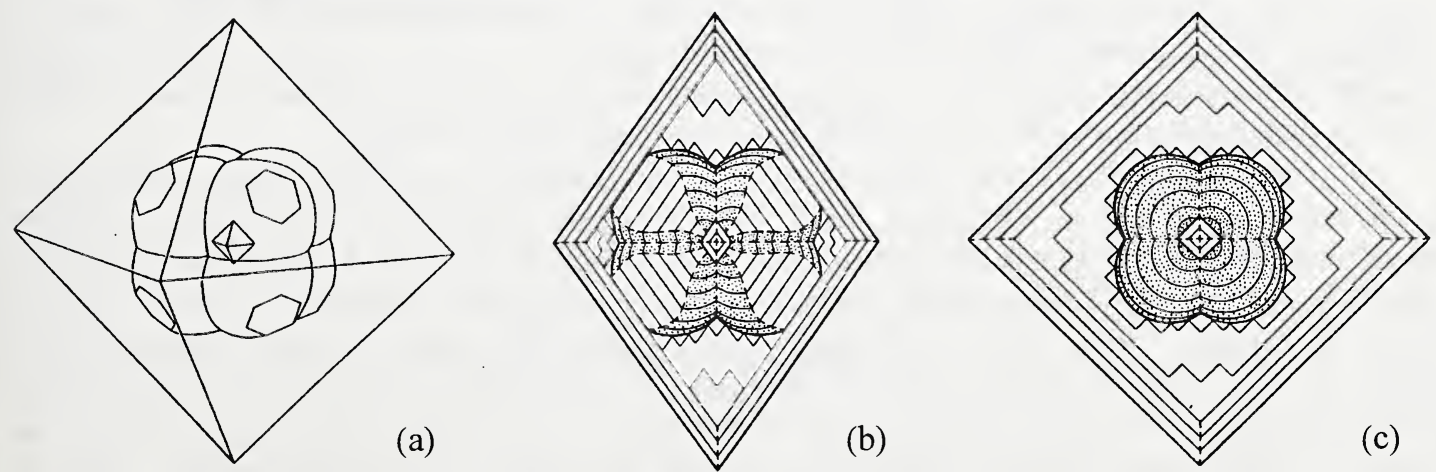

Fig.3. Internal morphology model of a natural diamond of octahedral habit (growth rate of the rounded form at the second stage is variable): (a) axonometric view; (b),(c) central sections parallel to (110) and (100). 